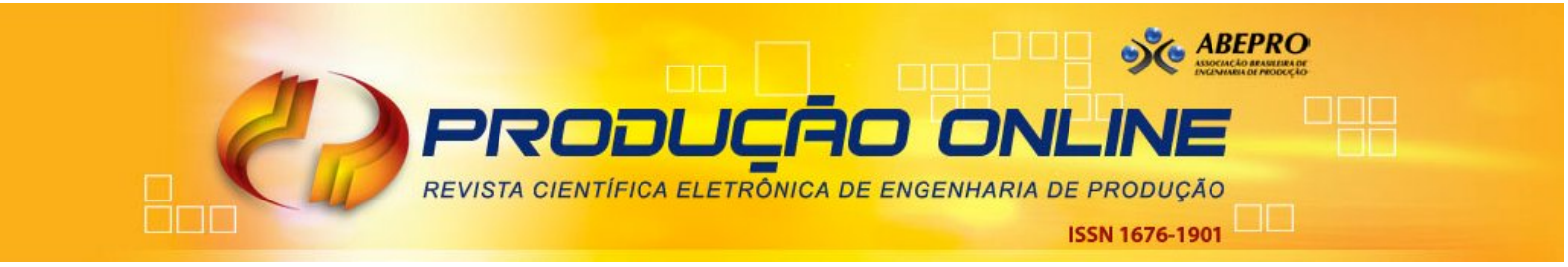

\title{
QUALIDADE DE VIDA E ESTRESSE OCUPACIONAL EM TRABALHADORES DE PRESÍDIOS ${ }^{1}$
}

\section{LIFE QUALITY AND OCCUPATIONAL STRESS IN PRISONS WORKERS}

\author{
Aurea Luzia Carvalho Fernandes* E-mail: aurealuzia@hotmail.com \\ Valéria Lira de Sousa* E-mail: walerialira@gmail.com \\ André Luiz Dantas Bezerra** E-mail: dr.andreldb@gmail.com

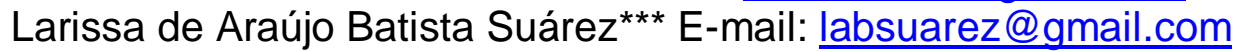 \\ Vandezita Dantas de Medeiros Mazzaro*** E-mail: vanmazzaro@hotmail.com

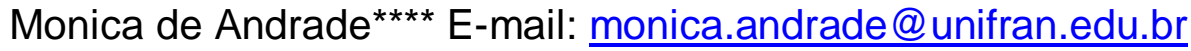

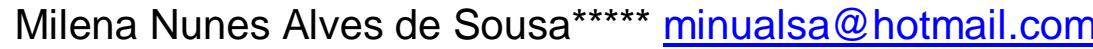 \\ *Faculdade Santa Maria (FSM), Cajazeiras, PB \\ ${ }^{*}$ Centro Universitário de João Pessoa (UNIPÊ), João Pessoa, PB

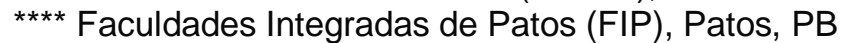 \\ **** Universidade de Franca (UNIFRAN), São Paulo, SP

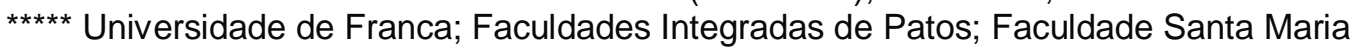

\begin{abstract}
Resumo: O Sistema Penitenciário brasileiro apresenta muitas falhas, que envolvem desde o tratamento dispensado aos presos, como a situação de trabalho dos profissionais atuantes neste cenário. Este fato conduziu à realização desta investigação, a qual teve o propósito de identificar à presença de estresse decorrente das atividades laborais, avaliar o nível de qualidade de vida dos trabalhadores e correlacionar as dimensões de qualidade de vida com o estresse ocupacional entre os dos agentes prisionais. Pesquisa descritiva, transversal e quantitativa, realizada com 35 trabalhadores (70\% do universo) atuantes no sistema carcerário de Cajazeiras, Paraíba após parecer 815.685 do Comitê de Ética em Pesquisa da Faculdade Santa Maria. Para a coleta de dados foram utilizados o World Health Organization Quality of Life - Bref (WHOQOL-Bref) e a Job Stress Scale (JSS). A análise foi feita a partir da estatística descritiva e inferencial. Nas comparações entre os domínios de qualidade de vida e os fatores de estresse no trabalho, utilizou-se o teste de Friedman e para controlar o efeito do erro de conjunto realizaram-se testes de bonferroni. Os resultados, quanto ao estresse ocupacional, indicaram que a demanda psicológica foi o que apresentou maior pontuação $(3,74)$ e a falta de controle do trabalho a menor $(2,71)$. O nível de qualidade de vida total dos agentes prisionais apresentou média de 71,07, com maior escore para o domínio social (76,90 pontos) e o menor ao ambiental (62,23 pontos). Ao correlacionar a qualidade de vida com o estresse, houve correlação negativa e significativa da qualidade de vida total com a falta de controle sobre o trabalho $(\rho=-0,42 ; p<0,05)$ e com o estresse total $(\rho=-0,41 ; p<0,05)$ mostrando que quanto maiores os níveis de estresse menor a qualidade de vida. Apesar de a qualidade de vida ter sido adequada, o domínio ambiental tem implicado em sua redução, bem como o estresse ocupacional, sendo este mais afetado pela falta de controle do trabalho. Sugerem-se intervenções no campo da promoção da saúde e segurança no ambiente ocupacional, com melhorias gerais nas condições de vida e de trabalho dos agentes prisionais.
\end{abstract}

Palavras-chave: Trabalho. Presídio. Qualidade de Vida. Estresse Ocupacional.

\footnotetext{
${ }^{1}$ Artigo originado a partir de trabalho de conclusão de curso em enfermagem pela Faculdade Santa Maria, Cajazeiras-PB, Brasil, desenvolvida sem fontes de financiamento, sem conflitos de interesse e aprovado pelo Comitê de Ética em Pesquisa da pela Faculdade Santa Maria, parecer: 815.685.
} 


\begin{abstract}
The Brazilian penitentiary system has many flaws, which involve the treatment of the prisoners, and also the work situation of the professionals in this setting. This fact led to this investigation, which had as a purpose to identify the presence of stress due to labor activities, assess the quality of life of the workers and correlate the dimensions of quality of life to occupational stress among the prison officers. This is a descriptive, transversal and quantitative research , carried out with 35 workers $(70 \%$ of the universes ) working in the prison system of the municipality of Cajazeiras, Paraiba after the approval of the Research Ethics Committee of the Faculty of Santa Maria (protocol number 815.685). For the data collection the World Health Organization Quality of Life - Bref (WHOQOL-Bref) and the Job Stress Scale (JSS) were used. The data was analyzed through the descriptive and inferential statistics. In the comparison between the quality of life and work-related stress domains, the Friedman test was used and to control the error effect Bonferroni's test was carried out. The results, regarding occupational stress, indicated that the psychological demand was what presented the highest score (3.74) and lack of control over work the lowest one (2.71). The total level of quality of life of the prison officers presented an average of 71.07, with the highest score being for the social domain (76.90 points) and the lowest score for the environmental domain (62.23 points). When correlating the quality of life with stress, there was a negative and significant correlation of the total quality of life with the lack of control over work $(\rho=-0.42 ; p<0.05)$ and with the total stress $(\rho=-$ $0.41 ; p<0.05)$ showing that the higher the levels of stress, lower the quality of life level. Despite the quality of life having been adequate, the environmental domain has led to its reduction, as well as the occupational stress, being this factor more affected by the lack of control over work. Interventions in the field of promotion of occupational health and safety, with general improvements in the life and work conditions of the prison agents are suggested.
\end{abstract}

Keywords: Work. Prison. Quality of Life. Occupational Stress.

\title{
1 INTRODUÇÃO
}

A segurança pública é uma das temáticas mais relevantes da atualidade, pois tem crescido a violência nos grandes centros urbanos, bem como em cidades interioranas. Apesar disto, o Sistema Penitenciário brasileiro apresenta muitas falhas, que envolvem desde o tratamento dispensado aos presos, como a situação de trabalho dos profissionais atuantes neste cenário, marcada pela superlotação das prisões, conflitos internos, número insuficiente de trabalhadores para atender a demanda e complexidade da organização (MENNOIAL et al., 2014).

Como é um sistema social composto por um grupo de pessoas submetidas a um regime de controle total, o poder empregado no sistema penitenciário é o uso da força física, uma vez que a proposta de tratamento penal visa reeducar o apenado para que o mesmo possa ser reinserido socialmente. A contradição entre a proposta de tratamento teoricamente implicada e o cotidiano de violência a que estão fadados, conduz para uma dialética conversiva que vai refletir diretamente nos agentes prisionais, que buscam garantir a segurança interna e a ordem entre os detentos nos presídios, e os quais dividem o mesmo espaço físico antagônico de ideias e valores (BONEZ; MORO; SEHNEM, 2013; SALLA, 2006). 
O quadro faz alusão aos riscos ocupacionais inerentes ao processo de trabalho no Sistema Penitenciário em que, pela exposição a eles, problemas são vivenciados pelos trabalhadores de presídios, atuando sobre seu sofrimento. Para Tschiedel; Monteiro (2013), as precárias condições de trabalho são fatores provocadores de sofrimento mental no grupo.

Saldaña; Salas-Menotti; Solórzano (2007) reforçam a problemática psicossocial, ao lidarem com homicídios, suicídios, acidentes e uso de sustâncias psicoativas. Corroboram com a afirmativa Bevan; Houdmont; Menear (2010). Tais autores verificaram que as condições psicossociais do trabalho podem estar relacionadas com os resultados de estresse entre os agentes prisionais, com efeitos negativos sobre o seu bem estar.

Bonez; Moro; Sehnem (2013) avigoram que estes profissionais estão propensos aos agravos, especialmente, pelas diversas situações laborais estressoras e redutoras de sua qualidade de vida. Paralelamente, Mennoial et al. (2014) destacam a exposição aos agentes biológicos (microorganismos em geral) e o estresse ocupacional, sendo estes, os principais riscos decorrentes das tarefas e das atribuições dos mencionados trabalhadores.

Embora a literatura referencie o cenário crítico vivenciado pelos agentes do Sistema Penitenciário, o que se percebe é a escassez de abordagens que contemplem esta população trabalhadora. Tschiedel; Monteiro (2013) dizem que a categoria é invisível, mesmo no Brasil com o crescimento vertiginoso de indivíduos privados de liberdade e com a realidade carcerária demarcada por condições de precariedade. Bonez; Moro; Sehnem (2013, p. 508), na direção destas considerações, asseveram que "são raros os estudos que se preocupam com a saúde desses agentes responsáveis pela segurança penitenciária".

Pelas proposituras e especificidades do processo de trabalho da categoria, parece oportuno abordar o nível de estresse ocupacional entre os agentes de segurança penitenciária e a sua qualidade de vida. Esta pode ser definida como um conjunto subjetivo e multidimensional de impressões positivas e negativas que cada ser humano possui, sendo simultaneamente um produto de diversos fatores que 0 afetam e um processo experimentado a cada momento. A boa qualidade de vida é 
aquela que oferece um mínimo de condições para que as pessoas possam desenvolver o máximo de suas potencialidades (ANDUJAR, 2006).

Quanto ao estresse ocupacional, pode-se considerá-lo como situações ameaçadoras da satisfação profissional, da saúde física e da mental, percebidas pelo trabalhador no ambiente ocupacional (MENDOZA; MEDEIRO; COSTA, 2007). Os autores reforçam que os fatores estressores são capazes de prejudicar a interação do profissional com o próprio ambiente laboral quanto com os colegas de trabalho.

Suas consequências não se limitam ao próprio indivíduo estressado. Para Correia (2006, p. 40), "o estresse do trabalho interfere significativamente nas relações de afeto e amizade podendo inclusive desencadear outros problemas ainda mais estressantes gerando um círculo vicioso de proporções cada vez mais imprevisíveis".

Pela compreensão da relevância desta pesquisa, surgiram como questões norteadoras: Há presença de estresse ocupacional entre os agentes prisionais? Qual o nível de qualidade de vida destes trabalhadores? O estresse relacionado ao trabalho tem implicações na qualidade de vida do grupo? Afinal, a carga de trabalho pode refletir em desgaste do trabalhador, quer seja no âmbito físico, psíquico e/ou mental (MOTTER; SANTOS; GUIMARÃES, 2015).

Partindo das explicitações anteriores, propõe-se com esta investigação identificar à presença de estresse decorrente das atividades laborais, avaliar o nível de qualidade de vida dos trabalhadores e correlacionar as dimensões de qualidade de vida com o estresse ocupacional entre os dos agentes prisionais, a fim de identificar possíveis implicações de ambos sobre a categoria, como forma de subsidiar propostas de intervenções no campo da prevenção de agravos e da promoção de saúde, a partir da implantação de programas de qualidade de vida no trabalho.

\section{MATERIAIS E MÉTODOS}

Pesquisa descritiva, transversal, com abordagem quantitativa. A investigação descritiva contempla a caracterização da população ou fenômeno em análise 
(FIGUEIREDO, 2008), o estudo transversal envolve "a coleta de dados em determinado ponto temporal (POLIT; BECK, 2011, p. 268), e a quantitativa demanda o uso da quantificação, se apropriando dos procedimentos estatísticos para quantificação dos resultados (FIGUEIREDO, 2008).

O estudo ocorreu com os agentes penitenciários do presídio masculino de segurança máxima (Presídio Padrão Regional) do município de Cajazeiras, Paraíba, cidade situada no alto sertão paraibano e em pleno semiárido nordestino. A população era de 50 profissionais atuantes no presídio outrora citado, destes, 35 participaram do estudo (70\%), sendo 30 do sexo masculino e cinco do feminino.

A amostra foi definida mediante elementos não probabilísticos, a partir de critérios de inclusão e exclusão pré-definidos. Inclusão: ser agente prisional atuante; ter no mínimo seis meses de experiência na área; aceitar participar do estudo voluntariamente, assinando o Termo de Consentimento Livre e Esclarecido (TCLE). Exclusão: estar de licença médica no período destinado à coleta de dados realizada no período de agosto e setembro de 2014, após a aprovação do projeto pelo Comitê de Ética em Pesquisa (CEP) da Faculdade Santa Maria (FSM), conforme CAAE 32556114.8.0000.5180/nº do parecer: 815.685.

A coleta de dados foi realizada com tempo estimado de aproximadamente 20 minutos por agente e no próprio local de trabalho. Para tanto, houve a aplicação de dois questionários validados no Brasil. Para mensurar a qualidade de vida foi adotado o World Health Organization Quality of Life - Bref (WHOQOL-Bref), composto por 26 questões (FLECK et al., 2000). Para os autores, esta versão preserva a abrangência do termo qualidade de vida por incluir itens referentes aos aspectos físicos, psicológicos, ao meio ambiente e quanto às relações sociais. $\mathrm{Na}$ avaliação do estresse no trabalho utilizou-se a versão resumida da Job Stress Scale (JSS), que contém 17 questões, com cinco delas úteis para avaliar a demanda psicológica no trabalho, seis o controle sobre o trabalho e seis referentes ao apoio social (URBANETTO et al., 2011).

Os dados foram tabulados e analisados no Statistical Package for Social Sciences (SPSS), versão 20.0. Utilizou-se de análises descritivas de tendência central (média e mediana), de dispersão (desvio-padrão-DP e valores máximos e mínimos) e frequências e porcentagem. Para fazer comparações entre os domínios 
de qualidade de vida e os fatores de estresse no trabalho, utilizou-se o teste de Friedman (teste utilizado para realizar comparações entre medidas repetidas com distribuição na paramétrica), para controlar o efeito do erro de conjunto (existente por conta das múltiplas comparações) realizaram-se testes de Bonferroni. Os dados foram apresentados em tabelas. Aceitou-se como significativo um erro de até $5 \%$, ou seja, $p \leq 0,05$.

\section{RESULTADOS E DISCUSSÃO}

Tabela 1 - Descrição e comparação dos domínios de qualidade de vida

\begin{tabular}{lccccc}
\hline \multicolumn{1}{c}{ Variáveis } & Média & DP & Mediana & Mínimo & Máximo \\
& & & & & \\
\hline Domínio Físico & 73,97 & 14,71 & $78,57 \mathrm{a}$ & 46,43 & 100,00 \\
Domínio Psicológico & 75,00 & 11,02 & $75,00 \mathrm{a}$ & 54,17 & 100,00 \\
Domínio Social & 76,90 & 10,51 & $75,00_{\mathrm{a}}$ & 50,00 & 100,00 \\
Domínio Ambiental & 62,23 & 9,01 & $62,50_{\mathrm{b}}$ & 43,75 & 84,38 \\
Qualidade de Vida Geral & 71,07 & 13,48 & 75,00 & 37,50 & 100,00 \\
\hline
\end{tabular}

Fonte: Dados da Pesquisa (2014)

Nota 1: Teste de Friedman ( $p$-valor) $<0,001$.

Nota 2: a diferente significativamente $(p \leq 0,05)$ de $b$ (teste de bonferroni)

A média, mediana, desvio-padrão, valores mínimo e máximo referentes a cada domínio do WHOQOL-Bref estão descritos na Tabela 1. Em relação a comparação dos domínios de qualidade de vida dos participantes da pesquisa, os resultados mostraram que quando se utiliza a média, o Domínio que apresentou maior escore foi o social $(76,90)$, seguido pelos domínios psicológico $(75,00)$, físico $(73,97)$ e o ambiental $(62,23)$. Portanto, este último domínio foi o que recebeu pior avaliação.

Segundo Vasconcellos; Costa-Val (2008), o domínio ambiental é composto por facetas que abordam a segurança física, ambiente do lar, recursos financeiros, cuidados de saúde e sociais, oportunidade de adquirir novas informações e habilidades, recreação/lazer, ambiente físico e transporte.

O comprometimento no mencionado domínio era de se esperar, visto que o ambiente de trabalho dos agentes prisionais é marcado pela exposição cotidiana a incontáveis riscos (MENNOIAL et al., 2014; BONEZ; MORO; SEHNEM, 2013; TSCHIEDEL; MONTEIRO, 2013; REIS et al., 2012; BEVAN; HOUDMONT; 
MENEAR, 2010; MENDOZA; MEDEIRO; COSTA, 2007; SALDAÑA; SALASMENOTTI; SOLÓRZANO, 2007).

Correia (2006) ressalta que existem riscos importantes que podem afetar a qualidade de vida dos agentes penitenciários e os quais são inerentes à profissão e os quais refletem sobre sua saúde e segurança, mas não podem ser minimizados porque fazem parte da característica da função, a exemplo das atividades repetitivas e o convívio constante com a massa carcerária.

Embora com trabalhadores diferentes, os resultados se assemelham com os da pesquisa realizada por Vasconcellos; Costa-Val (2008) no município de Lagoa Santa/MG, no qual a média dos escores acima de 50 pontos e a ordem decrescente dos domínios pertencentes ao WHOQOL-Bref encontrados foram: Físico - 82,8 pontos; Relações Sociais - 77 pontos; Psicológico - 76 pontos e Meio Ambiente 59,5 pontos.

O WHOQOL-Bref tem sido amplamente utilizado e estudos aplicaram o instrumento para mensurar a qualidade de vida em trabalhadores (LUCENA et al., 2015; CASTRO; HÖKERBERG; PASSOS, 2013; CALUMBI et al., 2010). "Alterações em um ou mais domínios podem implicar em alterações na qualidade de vida global" (PEREIRA, 2006, p. 34).

A qualidade de vida total, que obteve escore de 71,07 pontos, indicou um bom nível de qualidade de vida. O resultado considerado satisfatório pode relacionar-se com a importância atribuída ao trabalho pelos agentes prisionais, indicando satisfação no trabalho. Estudo qualitativo realizado por Santos; Santos (2011), com o propósito de analisar a qualidade de vida no ambiente de trabalho na Unidade Penitenciária Estadual de Ponta Grossa, Paraná, indicou que entre os 61 agentes participantes do estudo, 73\% apontaram que o trabalho desenvolvido na penitenciária é importante para sua realização pessoal, mostrando-se felizes com as atividades cotidianas e reconhecendo a relevância deste trabalho para a sociedade, visto que tenta combater a margilinalidade e a violência.

Apesar do achado geral e pelo fato de que cada dimensão afetada pode comprometer a qualidade de vida global, ressalta-se a importância de adotar um programa de qualidade de vida no trabalho, o qual tem o propósito de melhorar a satisfação e o bem estar do trabalhador, e por outro lado, aumentar a produtividade 
e o desempenho, visto como intimamente inter-relacionado ao nível de satisfação no ambiente de trabalho, ou seja, o alcance dos objetivos organizacionais está condicionado aos interesses individuais, principalmente quando se fala em um mercado tão competitivo (OLIVEIRA et al., 2013).

Conforme Correia (2006), a dinâmica penitenciária comporta mecanismos preventivos, não somente do ponto de vista da atividade intramuros como do desenvolvimento pessoal e profissional de uma maneira mais ampla. Conforme o autor, há fatores que interferem na qualidade de vida do agente prisional, na sua longevidade e expectativa de vida que poderiam ser equacionados, minimizados ou preventivamente tratados, por exemplo, numa perspectiva da Segurança e Medicina do Trabalho. Estes elementos se mostram na forma de reflexos na vida do agente penitenciário.

Quanto à descrição dos fatores desencadeantes de estresse no trabalho, os achados estão descritos na tabela 2.

Tabela 2 - Descrição dos fatores de estresse no trabalho

\begin{tabular}{lccccc}
\hline \multicolumn{1}{c}{ Variáveis } & Média & DP & Mediana & Mínimo & Máximo \\
\hline Demanda Psicológica & 3,74 & 0,41 & $3,83_{\mathbf{a}}$ & 2,50 & 4,67 \\
Controle sobre o & 2,71 & 0,43 & $2,66_{\mathbf{b}}$ & 2,00 & 3,33 \\
Trabalho & 2,99 & 0,40 & $3,16_{\mathbf{c}}$ & 2,17 & 3,83 \\
Apoio Social & 3,15 & 0,24 & 3,16 & 2,44 & 3,72 \\
Estresse Total & & & &
\end{tabular}

Fonte: Dados da Pesquisa (2014)

Nota 1: Teste de Friedman ( $p$-valor) $<0,001$.

Nota 2: "a", "b" e "c" diferem significativamente $(p \leq 0,05)$ (teste de bonferroni)

A tabela 2 faz uma descrição dos níveis de estresse entre a amostra pesquisada. Verifica-se que aquele proveniente da demanda psicológica foi o que apresentou significativamente maior pontuação $(3,74)$ e a falta de controle do trabalho foi o fator mais comprometido, com a menor pontuação $(2,71)$.

Esse resultado demonstra que o controle sobre o trabalho é o elemento que vem interferindo na possibilidade de desencadear o estresse (embora os agentes não tenham se apresentando estressados ainda) e, posteriormente, afetar a sua saúde mental, o que pode implicar no nível de qualidade de vida. Considerando o 
grupo de trabalhadores, Santos; Santos (2011) identificam que para estes indivíduos a saúde mental tem interferido no desenvolvimento de seu trabalho.

Por sua vez, diferentemente desta pesquisa, estudos (BASTOS et al., 2013; SILVA; SUÑE, 2011) apontaram que a demanda psicológica dos agentes prisionais tem sido o principal estressor devido a sua atividade laboral podem contribuir para o aparecimento de sintomas característicos do estresse.

Para Bastos et al. (2013), em investigação com 833 servidores penitenciários do Programa de Atenção Psicossocial dos Servidores Penitenciários, foi detectado que $72,75 \%$ dos entrevistados declararam que seu trabalho era estressante; em pergunta, na qual o servidor poderia apontar mais de um sintoma que tem apresentado nos últimos meses, acreditando ele estarem associados ao seu trabalho, 310 afirmaram-se ansiosos, 272 com alterações no sono, 263 com dores musculares, 243 com alterações de humor, 228 agitados e 126 disseram que se irritam constantemente, entre outros sintomas listados.

Já em outro estudo feito por Silva; Suñe (2011), que buscou verificar os níveis de estresse e atividade física dos Agentes Penitenciários Administrativos que trabalham nas Casas Prisionais e no Órgão Central da Superintência de Serviços Penitenciários (SUSEPE), não foram constatados altos níveis de estresse no trabalho em nenhuma das populações investigadas, porém foi detectada elevada demanda psicológica que poderia configurar-se como um elemento estressor, se outros fatores como o nível de controle e de apoio social não tivessem se apresentado em níveis semelhantemente altos.

Refletindo sobre os aspectos mais críticos entre os elementos estressores, a falta de controle no trabalho parece característica do processo laboral. Para Reis et al. (2012) existem riscos psicossociais associados às responsabilidades de manter a ordem na prisão pois pode ser agredido e ser contaminado por doenças infecciosas. De modo colaborativo, Campos; Sousa (2011), afirmam que o adoecimento e o estresse podem surgir da dificuldade que o agente tem em lidar com determinadas atitudes dos detentos, fazendo-os sentirem-se mortificados, humilhados e desafiados. Diante de situações como esta, os autores destacam que tais trabalhadores despendem uma energia ao tentar se equilibrar entre os dois mundos 
em que vive - intra e extramuros -; e ainda, enquanto obedecedor de ordens e impositor destas.

Acrescentam Morse et al. (2011) que são implicações da variável controle no trabalho, a falta de segurança nas atividades, os requisitos administrativos e os atributos próprios do trabalho. E para Bonez; Dal Moro; Sehnem (2013, p. 509) "a ansiedade causada pelo fato de o agente penitenciário estar diariamente submetido a situações de pressão e desconforto" é outro fator relevante.

Conforme Ghaddar; Ronda; Nolasco (2011), o ambiente ocupacional nos presídios é psicologicamente exigente. A partir de pesquisa Bonez; Dal Moro; Sehnem (2013), Reis et al. (2012) e Morse et al. (2011) constataram que os níveis de estresse dos agentes são elevados, o que tem refletido em doenças de vários tipos como a hipertensão arterial sistêmica, além de implicações negativas sobre o relacionamento familiar.

Foram realizadas também, correlações entre a qualidade de vida e o estresse dos agentes prisionais (tabela 3).

Tabela 3 - Correlações entre a qualidade de vida e o estresse no trabalho

\begin{tabular}{lcccc}
\hline \multicolumn{1}{c}{ Variáveis } & $\begin{array}{c}\text { Demanda } \\
\text { psicológica }\end{array}$ & $\begin{array}{c}\text { Controle sobre } \\
\text { o trabalho }\end{array}$ & Apoio social & Estresse total \\
\hline Domínio Físico & $-0,16$ & $-0,05$ & $-0,09$ & $-0,17$ \\
Domínio Psicológico & $-0,32$ & $-0,04$ & 0,05 & $-0,17$ \\
Domínio Social & $-0,29$ & $-0,03$ & $-0,16$ & $-0,27$ \\
Domínio Ambiental & $-0,32$ & 0,13 & 0,15 & $-0,01$ \\
Qualidade de Vida Geral & $-0,13$ & $-\mathbf{0 , 4 2}$ & $-0,16$ & $\mathbf{- 0 , 4 1}^{*}$ \\
\hline
\end{tabular}

Fonte: Dados da Pesquisa (2014)

Considerando as correlações entre a qualidade de vida e o estresse no trabalho, observou-se que houve correlação negativa e significativa da qualidade de vida global com a falta de controle sobre o trabalho $(\rho=-0,42 ; p<0,05)$ e com o estresse total $(\rho=-0,41 ; p<0,05)$ mostrando que quanto maiores os níveis de estresse menor a qualidade de vida.

Pesquisa canadense, a partir de revisão sistemática da literatura, constatou que existem cinco categorias de estressores organizacionais entre os agentes prisionais, sendo elas: estressores inerentes ao cargo, a função na organização, recompensas, relações de supervisão no local de trabalho e da estrutura, bem como o clima organizacional (FINNEY et al., 2013). Os autores também afirmam que a 
estrutura e o clima organizacional parecem ter o maior impacto sobre os níveis de estresse ocupacional e o desenvolvimento da síndrome de burnout.

Para Reis et al. (2012) é possível que o estresse e a insegurança que acometem os agentes também aconteçam pelo fato de que, além das regras formais e dos papéis oficialmente estabelecidos, as instituições carcerárias também são conduzidas à base de regras informais, que subvertem e, às vezes, suplantam as regras formais, sem as quais administrar a instituição ou trabalhar no seu meio interno seria quase que impossível.

Silva; Suñe (2011), ao analisar o estresse como um todo, bem como verificando as altas pontuações das dimensões controle e apoio social somadas, a fim de relacionar com a alta demanda psicológica, acabou por indicar um equilíbrio, diminuindo a tendência ao estresse, pois apesar do servidor estar sobre forte carga de trabalho mental, existe uma boa relação com colegas e ambiente e a possibilidade de optar sobre suas ações.

Para algumas profissões, o estresse no trabalho é neutralizado pelo prestígio e pelo reconhecimento social, porém, o trabalho do agente de presídios oferece poucas compensações desse tipo. O estresse, a rotina e os horários de trabalho são fatores negativos da atividade profissional desses profissionais, e tais aspectos têm impactos extremamente duros sobre seu moral, com conseqüente redução de sua qualidade de vida no trabalho.

Pelas proposituras, há de convir à necessidade de intervenções no campo da segurança e saúde no trabalho, embora os resultados encontrados nesta pesquisa tenham apresentado limitações quanto ao tipo de estudo (transversal), bem como pela falta de garantias referentes à veracidade das informações fornecidas pela população-alvo, bem como pelo fato de a amostra não contemplar todo o universo populacional (70\%).

\section{CONSIDERAÇÕES FINAIS}

Os achados desta pesquisa realizada com os agentes penitenciários do presídio masculino de segurança máxima (Presídio Padrão Regional) do município de Cajazeiras, Paraíba, indicaram positividade e negatividade em muitos aspectos. 
Deste modo, destaca-se que os três objetivos propostos no estudo foram alcançados.

Inicialmente, ao contemplar os fatores de estresse no trabalho, os resultados propuseram que a falta de controle no trabalho foi o pior escore apresentado, corroborando com o aumento do nível de estresse entre os agentes prisionais.

Referindo-se a qualidade de vida, os achados alvitraram que os agentes prisionais apresentaram bons escores de qualidade de vida para quase todos os domínios do WHOQOL-Bref quando comparadas à pontuação máxima dos escores para cada domínio e às médias encontradas para os diferentes domínios da qualidade de vida na população estudada, contudo, foi possível verificar que o pior escore foi para o domínio ambiental e o melhor para o social. Destaca-se, ainda, que o nível de qualidade de vida global apresentou-se satisfatório, uma vez que a média de pontos foi superior a 70 .

Ainda, ao correlacionar a qualidade de vida e com o estresse entre os trabalhadores estudados, constatou-se haver correlação negativa e significativa entre a qualidade de vida total com a falta de controle sobre o trabalho e com o estresse total, mostrando que, quanto maiores os níveis de estresse menor a qualidade de vida.

Considerando os achados, sugerem-se intervenções no campo da promoção da saúde e segurança no ambiente ocupacional, com melhorias gerais nas condições de vida e de trabalho dos agentes prisionais.

\section{REFERÊNCIAS}

ANDUJAR, A. M. Modelo de qualidade de vida dentro dos domínios biopsicosocial para aposentados. 2006. $280 \mathrm{f}$. Tese (Doutorado em Engenharia de Produção) - Universidade Federal de Santa Catarina, Florianópolis, 2006. Disponível em:

<http://repositorio.ufsc.br/bitstream/handle/123456789/88517/229433.pdf?sequence =1\&isAllowed=y>. Acesso em: 23 set. 2014.

BASTOS, F. B. et al. Atenção psicossocial do servidor penitenciário. In: VI CONGRESSO CONSAD DE GESTÃO PÚBLICA, Anais... Brasília/DF, abr. 2013. Disponível em: <http://consadnacional.org.br/wp-content/uploads/2013/05/168ATEN\%C3\%87\%C3\%83O-PSICOSSOCIAL-DO-SERVIDORPENITENCI\%C3\%81RIO.pdf>. Acesso em: 02 dez. 2014. 
BEVAN, A.; HOUDMONT, J.; MENEAR, N. The Management Standards Indicator Tool and the estimation of risk. Occup Med (Lond), v. 60, n. 7, p. 525-31, 2010. http://dx.doi.org/10.1093/occmed/kqq109

BONEZ, A.; MORO, E. D.; SEHNEM, S. B. Saúde mental de agentes penitenciários de um presídio catarinense. Psicol. Argum, v.31, n.74, p. 507-17, 2013.

http://dx.doi.org/10.7213/psicol.argum.31.074

CALUMBI, R. A. et al. Evaluation of the quality of life of anesthesiologists in the city of Recife. Rev Bras Anestesiol., v. 60, p. 42-5, 2010.

http://dx.doi.org/10.1590/S0034-70942010000100005

CAMPOS, J. C.; SOUSA, R. R. O adoecimento psíquico do agente penitenciário e o sistema prisional: estudo de caso - Sete Lagoas. In: XXXV ENCONTRO DA ANPAD, Anais... Rio de Janeiro/RJ, set. 2011. Disponível em:

<http://www.anpad.org.br/admin/pdf/GPR1760.pdf>. Acesso em: 02 dez. 2014.

CASTRO, M. M. L. D.; HOKERBERG, Y. H. M.; PASSOS, S. R. L. Validade dimensional do instrumento de qualidade de vida WHOQOL-BREF aplicado a trabalhadores de saúde. Cad. Saúde Pública, v. 29, n. 7, p. 1357-69, 2013. http://dx.doi.org/10.1590/S0102-311X2013000700010

CORREIA, A. P. Uma análise dos fatores de risco da profissão do agente penitenciário: contribuições para uma política de segurança e saúde na gestão penitenciária. 2006. 65 f. Monografia (Especialização em Gestão Penitenciária) Departamento de Ciências Sociais, Universidade Federal do Paraná, Curitiba, 2006. Disponível em:

<http://www.depen.pr.gov.br/arquivos/File/ADEMILDO_\%20PASSOS_CORREIA200 6.pdf>. Acesso em: 14 out. 2014.

FIGUEIREDO, N. M. A. Metodologia: interpretando autores. In: Método e metodologia na pesquisa científica. 3. ed. São Caetano do Sul: Yendis, 2008. p. 91-118.

FINNEY, C. et al. Organizational stressors associated with job stress and burnout in correctional officers: a systematic review. BMC Public Health, v. 13, n. 82, 2013. http://dx.doi.org/10.1186/1471-2458-13-8

FLECK, M. P. A et al. Aplicação da versão em português do instrumento abreviado de avaliação da qualidade de vida "WHOQOL-bref". Rev. Saúde Pública, v. 34, n. 2, p. 178-183, 2000. http://dx.doi.org/10.1590/S0034-89102000000200012

GHADDAR, A.; RONDA, E.; NOLASCO, A. Work ability, psychosocial hazards and work experience in prison environments. Occup Med (Lond), v. 61, n. 7, p. 503-8, 2011. http://dx.doi.org/10.1093/occmed/kgr124 
LUCENA, K. L. Sofrimento psíquico e qualidade de vida entre policiais militares do batalhão de operações especiais. Revista Interdisciplinar em Saúde, v. 2, n. 1, p. 157-76, 2015. Disponível em:

<http://www.interdisciplinaremsaude.com.br/Volume_3/Trabalho_10.pdf>. Acesso em: 28 jul. 2015.

MENDOZA, R.; MEDEIRO, V.; COSTA, J. B. Comprometimento organizacional, fatores estressantes do trabalho e identidade social: um estudo exploratório. Revista Ciências Humanas e Artes, v. 13, n. 1, p. 92-100, 2007. Disponível em: $<$ http://www.ch.ufcg.edu.br/arius/01_revistas/v13n1/09_arius_13_1_comprometiment o_organizacional_fatores_estressantes_do_trabalho_e_identidade_social.pdf $>$. Acesso em: 14 out. 2014.

MENNOIAL, N. V. et al. Occupational risk factors and medical prevention in corrections officers. G Ital Med Lav Ergon., v. 36, n. 4, p. 405-9, 2014. Disponível em: <http://www.ncbi.nlm.nih.gov/pubmed/25558744>. Acesso em: 28 jul. 2015.

MORAES, P. R. B. A identidade e o papel de agentes penitenciários. Tempo Social, revista de sociologia da USP, v. 25, n. 1, p. 131-147, 2005. Disponível em: <http://www.scielo.br/pdf/ts/v25n1/07.pdf>. Acesso em: 02 dez. 2014.

MORSE, T. et al. Talking about health: correction employees' assessments of obstacles to healthy living. J Occup Environ Med., v. 53, n. 9, p. 1037-45, 2011. http://jcx.sagepub.com/content/17/3/208

MOTTER, A. A.; SANTOS, M.; GUIMARÃES, A. T. B. O que está a sombra na carga de trabalho de estivadores? Revista Produção On Line, v. 15, n. 1, p. 321-44, 2015. http://dx.doi.org/10.14488/1676-1901.v15i1.1845

OLIVEIRA, J. S. et al. Qualidade de vida no trabalho: um estudo sobre a avaliação por agentes de segurança penitenciária. RaUnp, v. 5, n. 2, abr./set. 2013. Disponível em: <http://www.spell.org.br/documentos/download/10566>. Acesso em: $01 \mathrm{dez}$. 2014.

PEREIRA, R. J. et al . Contribuição dos domínios físico, social, psicológico e ambiental para a qualidade de vida global de idosos. Rev. psiquiatr. Rio Gd. Sul, v. 28, n. 1, p. 27-38, 2006. http://dx.doi.org/10.1590/S0101-8108200

POLIT, D. F.; BECK, C. T. Delineamento de pesquisas quantitativas. In: Fundamentos da pesquisa em enfermagem: avaliação de evidências para a prática da enfermagem. 7. ed. Porto Alegre: Artmed, 2011. p. 249-87.

REIS, J. F. G. et al. Agentes prisionais: Percepções e conflitos de uma profissão de risco. In: XV CISO ENCONTRO NORTE E NORDESTE DE CIÊNCIAS SOCIAIS, Anais... São Paulo, 2012. Disponível em: <http://www.sinteseeventos.com.br/ciso/anaisxvciso/resumos/GT25-12.pdf> Acesso em: 02 dez. 2014. 
SALDAÑA, Á. C. T.; SALAS-MENOTTI, I; SOLÓRZANO, C. Descripción de las estadísticas de problemáticas psicosociales en guardianes penitenciarios de Colombia. Suma psicol., v. 14, n. 1, p. 7-22, 2007.

http://dx.doi.org/10.14349/sumapsi2007.35

SALLA, F. As rebeliões nas prisões: novos significados a partir da experiência brasileira. Rev. Sociologias, v. 8, n. 16, p. 274-307, 2006. Disponível em: <http://www.scielo.br/pdf/soc/n16/a11n16.pdf>. Acesso em: 20 jun. 2014.

SANTOS, M. P.; SANTOS, J. C. P. Qualidade de vida no ambiente de trabalho: um estudo de caso na unidade penitenciária estadual de ponta grossa - Paraná.

Revista Jurídica, v. 15, n. 30, p. 21-38, 2011. Disponível em: <http://proxy.furb.br/ojs/index.php/juridica/article/view/2857/2013>. Acesso em: 20 jun. 2014.

SILVA, T. A.; SUÑE, F. R. Nível de estresse e atividade física em agentes penitenciários administrativos da SUSEPE - 10ª DPR. 2011. Disponível em: <http://www.susepe.rs.gov.br/upload/1374841035_ARTIGO\%20\%20Nivel\%20de\%20Estresse $\% 20$ e\%20Atividade\%20Fisica\%20em\%20Agentes $\% 20$ Penitenciarios\%20Administrativos\%20da\%20SUSEPE\%20_\%2010a\%20DPR\%20po r\%20TIAGO\%20AMARAL\%20DA\%20SILVA\%5B1\%5D.pdf> Acesso em: 01 dez. 2014.

TSCHIEDEL, R. M.; MONTEIRO, J. K. Prazer e sofrimento no trabalho das agentes de segurança penitenciária. Estud. psicol. (Natal), v. 18, n. 3, p. 527-35, 2013. http://dx.doi.org/10.1590/S1413-294X2013000300013

URBANETTO, J. S. et al. Estresse no trabalho da enfermagem em hospital de pronto-socorro: análise usando a Job Stress Scale. Rev. Latino-Am. Enfermagem, Ribeirão Preto, v. 19, n. 5, p. 1-10, set./out. 2011. http://dx.doi.org/10.1590/S0104$\underline{11692011000500009}$

VASCONCELLOS, N. P. C.; COSTA-VAL, R. Avaliação da qualidade de vida dos agentes comunitários de Saúde de Lagoa Santa - MG. Rev. APS, v. 11, n. 1, p. 1728, jan./mar. 2008. Disponível em: <http://www.ufjf.br/nates/files/2009/12/017028.pdf>. Acesso em: 01 dez. 2014.

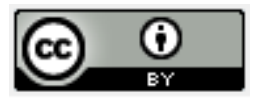

Artigo recebido em 28/07/2015 e aceito para publicação em 18/11/2015 DOI: $\underline{\text { http://dx.doi.org/ 10.14488/1676-1901.v16i1.2095 }}$ 